\title{
Improved properties of chemically modified graphene/poly(methyl methacrylate) nanocomposites via a facile in-situ bulk polymerization
}

\author{
X. Y. Yuan", L. L. Zou, C. C. Liao, J. W. Dai \\ College of Science, Chongqing Jiaotong University, 400074 Chongqing, China
}

Received 16 March 2012; accepted in revised form 17 May 2012

\begin{abstract}
The nanosheet of graphene was chemically modified by long alkyl chain for enhanced compatibility with polymer matrix and graphene/poly(methyl methacrylate) (PMMA) nanocomposites with homogeneous dispersion of the nanosheets and enhanced nanofiller-matrix interfacial interaction were fabricated via a facile in-situ bulk polymerization. The nanocomposites were characterized by X-ray diffraction, Fourier transform infrared spectroscopy, Scanning electron microscopy and thermogravimetry. The results showed that the graphene nanosheets were fully exfoliated in PMMA matrix and the thermal and mechanical properties of the nanocomposites were significantly improved at low graphene loadings. Large shifts of $15^{\circ} \mathrm{C}$ in the glass transition temperature and $27^{\circ} \mathrm{C}$ improvement of onset thermal degradation temperature were achieved with graphene loading as low as $0.07 \mathrm{wt} \%$. A $67 \%$ increase in tensile strength was also observed by the addition of only $0.5 \mathrm{wt} \%$ graphene. The method used in this study provided a novel route to other graphene-based polymers.
\end{abstract}

Keywords: nanocomposites, graphene, poly(methyl methacrylate), mechanical property, thermal property

\section{Introduction}

Graphene, a two-dimensional layer of $\mathrm{sp}^{2}$-bonded carbon atoms densely packed in a honeycomb crystal lattice, has attracted significant interests due to its exceptional mechanical, electrical, thermal and optical properties [1-6] and is being explored for use in a variety of applications such as electronics [7], composites [8, 9], catalysis sensors [10], transparent and flexible electrodes [11], solar cells and supercapacitor [12]. Among these potential applications, the use of graphene as one of promising nanofillers incorporating in a polymer matrix has been extensively investigated to develop cost-effective, high-performance graphene-polymer nancomposites (GPN) [13-15]. Recent studies have shown that polymer nanocomposites with graphene as nanofiller exhibit substantial property enhancements at much lower loadings than with other conventional nanofiller in many cases [13-16].

To achieve high-performance of GPNs, however, homogeneous dispersion of graphene nanosheets in the polymer hosts and proper interfacial interactions between the nanosheets and the surrounding matrix must be considered. The nature of graphene is not compatible with the organic polymer matrix and the individual sheets tend to restack owing to their large specific surface area and van der Waals interactions between the interlayers of graphene nanosheets, which lowers its effectiveness as a nanofiller for property improvements $[6,17]$. An effective way to overcome the agglomeration and enhance the compatibility between graphene nanosheets and polymer matrix is the surface modification of graphene via noncovalent and covalent interaction [14].

\footnotetext{
${ }^{*}$ Corresponding author, e-mail: yuanxy@cquc.edu.cn
}

(c) BME-PT 
Graphene oxide (GO), an important intermediate for preparing graphene via chemical reduction [18], consists of many oxygen-containing groups, such as hydroxyl, epoxy, carbonyl and carboxyl groups, which provide diverse active sites for covalent funcationalizations [17, 19]. Therefore, chemical interaction via direct coupling between polymers and the surface groups bound on GO sheets [20-26] and grafting some molecules including monomer [27] and atom transfer radical polymerization (ATRP) initiator [28-33] prior to polymerization could be accomplished. Pan et al. [25] prepared covalently functionalized graphene sheets by grafting a well defined thermo-responsive poly(N-isopropylacrylamide) via click chemistry and the nanocomposites exhibited a hydrophilic to hydrophobic phase transition at $33^{\circ} \mathrm{C}$ and a superior anticancer drug loading capacity of $15.6 \mathrm{wt} \%$. Fang et al. [30] covalently grafted an ATRP initiator onto the graphene sheet via a diazonium addition and the resulting polystyrene nanocomposites showed around 70 and $57 \%$ increases in tensile strength and Young's modulus with $0.9 \mathrm{wt} \%$ graphene nanosheets, respectively. By reacting Gamma-aminopropyltriethoxysilane-grafted graphene oxide sheets and maleic anhydride grafted polyethylene, Fang et al. [33] recently demonstrated a covalently bonded polyethylene grafted graphene oxide hybrid material with about $20 \%$ increase in Young's modulus at $0.3 \mathrm{wt} \%$ of graphene oxide and about $15 \%$ increase in yielding strength and tensile strength with only $0.1 \mathrm{wt} \%$ of graphene oxide were achieved. Chemical linkage between graphene nanosheets and polymer matrix could indeed enhance the interfacial adhesion, which favored the load transfer across the interface of the nanocomposites. However, current methods available for enhanced interfacial compatibility via covalent functionalization of graphene nanosheets with the polymer suffer from certain drawbacks, including tedious processes, rigorous reaction conditions, lack of precise control of polymer architecture and nanofiller content.

Compared to these aforementioned methods, noncovalent interaction between the nanofiller and polymer matrix via hydrogen bonding and $\pi-\pi$ stacking is more easily achieved by solution blending [3437], self assembly [38-40] and in situ interactive polymerization [41-44]. Liang et al. [34] demonstrated that molecular-level dispersion of graphene into poly(vinyl alcohol) by solution-mixing and the hydrogen bonding at the interface were key to $76 \%$ increase in tensile strength and $62 \%$ improvement of Young's modulus of the nanocomposites at only $0.7 \mathrm{wt} \%$ of GO Liu et al. [40] synthesized $\mathrm{pH}$-sensitive GPNs by attaching pyrene-terminated poly(2$\mathrm{N}, \mathrm{N}$-(dimethyl amino ethyl acrylate) and poly (acrylic acid) onto the basal plane of graphene sheets via $\pi-\pi$ stacking, respectively. Precipitation/extraction or solution casting to generate samples often coincide with these efforts, which causes an additional tedious work-up for purification. Therefore, it is very urgent to explore a facile and efficient method to compatibilize graphene sheets with the polymer hosts in GPNs while maintaining good dispersion of the nanosheets in the matrix.

In situ polymerization is an effective way to prepare layered-structure nanocomposite [45] and has been used to produce non-covalent graphene-based nanocomposites. Potts et al. [41] prepared chemically reduced graphene/poly(methyl methacrylate) (PMMA) by free radical solution-polymerization of methyl methacrylate (MMA) in the presence of exfoliated $\mathrm{GO}$ and an shift of over $15^{\circ} \mathrm{C}$ in the glass transition temperature with graphene loadings as low as $0.05 \mathrm{wt} \%$ and $28 \%$ increase of the elastic modulus at just $1 \mathrm{wt} \%$ loading were observed. To date, however, most of in situ polymerization techniques used for GPNs are focused on solution polymerization [41-44] and involve an intercalation of functionalized GO with a monomer prior to polymerization, where subsequent coagulation of polymer or reduction of GO are frequently involved. In addition, the tedious purification of GPNs by coagulation with non-solvent of the matrix polymer can potentially lead to aggregation of the nanofiller in the composites [46], which could worsen the properties of the resulting GPNs. Motivated by these results, in this work, we sought to explore a facile and efficient route for producing graphene/PMMA nanocomposites via in situ bulk-polymerization of MMA in the presence of Octadecylamine-modified graphene $\left(\mathrm{C}_{18}\right.$-graphene). The long-alkyl chain attached onto the edges of graphene favored homogeneous dispersion of graphene nanosheets in the matrix and good compatibility between graphene and PMMA. The obtained $\mathrm{C}_{18}$-graphene/PMMA nanocomposites exhibited significant enhancement in mechanical properties and thermal stability at 
very low loadings of graphene and the results are herein disclosed.

\section{Experimental}

\subsection{Materials}

Natural graphite flakes with an average particle size of $150 \mu \mathrm{m}$ and a purity of $>98 \%$ were supplied from Qingdao Nanshu Graphite Co., Ltd., China. Concentrated sulfuric acid (A.R., 98\%), hydrochloric acid (A.R., 36\%), Hydrogen peroxide (A.R., 30\%), Sodium nitrate (A.R.), N, N-dimethylformamide (DMF, A.R.) and ethanol (A.R.), were purchased from Chongqing Chuandong Chemical Reagent Factory, China. Potassium permanganate (A.R.) was purchased from Yixin Chemical Reagent Co., Ltd., China. Hydrazine hydrate (A.R., 85\%), Octadecylamine(A.R., ODA) and N, N-dicyclohexylcarbodiimide (DCC, A.R.) were provided from Sinopharm Chemical Reagent Co., Ltd., China. Methylmethacrylate (A.R.) and benzoyl peroxide (BPO, A.R.) were obtained from Kelong Chemical Reagent Factory, China and purified by distillation under reduced pressure. All the reagents were used as received without any further treatment.

\subsection{Preparation of graphene oxide (GO)}

GO was prepared according to the Hummers method [47]. Graphite (1.0 g), sodium nitrate $(1.0 \mathrm{~g})$ and concentrated $\mathrm{H}_{2} \mathrm{SO}_{4}(23 \mathrm{~mL})$ were mixed and cooled to $0^{\circ} \mathrm{C} . \mathrm{KMnO}_{4}(3.0 \mathrm{~g})$ was slowly added while stirring and the rate of addition was controlled to prevent the mixture temperature from exceeding $20^{\circ} \mathrm{C}$. After the complete addition of $\mathrm{KMnO}_{4}$, the mixture was then transferred to a $35^{\circ} \mathrm{C}$ water bath and stirred for $30 \mathrm{~min}$, during which a brownish-gray paste was formed. Next, $50 \mathrm{~mL}$ of deionized water was added to the solution, and the temperature was increased to $98^{\circ} \mathrm{C}$. After $15 \mathrm{~min}$, the solution was further treated with $100 \mathrm{~mL}$ of warm water and $10 \mathrm{~mL}$ of $30 \% \mathrm{H}_{2} \mathrm{O}_{2}$. The light-yellow warm solution was then filtered and the precipitate was washed with deionized water several times, and finally dried at $60^{\circ} \mathrm{C}$ under vacuum.

\subsection{Preparation of octadecylamine-modified graphene $\left(\mathrm{C}_{18}\right.$-graphene)}

Starting from GO, chemically reduced graphene (CRG) was prepared according to Ruoff's method
[48, 49]. For surface modification of CRG with long alkyl chain, $0.15 \mathrm{~g}$ of graphene was dispersed in $200 \mathrm{~mL}$ anhydrous DMF by $1 \mathrm{~h}$ of ulstrasonication (As2060B bath sonicator, Automatic Science Instrument Co. Ltd., China). 5.0 g of ODA and $2.0 \mathrm{~g}$ of DCC were then added and the mixture was stirred at $90^{\circ} \mathrm{C}$ under $\mathrm{N}_{2}$ for $48 \mathrm{~h}$. After filtration of the solution, the resulting solid was washed with excess ethanol and dried under vacuum.

\subsection{Preparation of octadecylamine-modified graphene/PMMA nanocomposites ( $\mathrm{C}_{18}$-graphene/PMMA)}

The original loading of $\mathrm{C}_{18}$-graphene was based on the weight of MMA monomer and $\mathrm{C}_{18}$-graphene/ PMMA nanocomposites were prepared via in situ bulk-polymerization. Typically, $0.02 \mathrm{~g}(0.1 \mathrm{wt} \%$ of MMA) of $\mathrm{C}_{18}$-graphene was added into $20.0 \mathrm{~g}$ of MMA and the mixture was treated with ultrasound at $0^{\circ} \mathrm{C}$ for $1 \mathrm{~h}$. The obtained black dispersion was then subjected to $15 \mathrm{~min}$ of centrifugation at $4000 \mathrm{rpm}$ to remove any unexfoliated agglomerate. The obtained $\mathrm{C}_{18}$-graphene/MMA suspension was purged with dry nitrogen for $10 \mathrm{~min}$ to remove traces of oxygen from the solution and $0.3 \mathrm{~g}$ of BPO was then added. Subsequently, the temperature was raised to $60^{\circ} \mathrm{C}$ to promote polymerization. The reaction was maintained at $60^{\circ} \mathrm{C}$ for $8 \mathrm{~h}$ until the mixture turned into transparent solid. A similar protocol was followed to prepare pure PMMA and $\mathrm{C}_{18}$-graphene/PMMA composites with original graphene loading of 0.1 , $0.5,1.0$ and $1.5 \mathrm{wt} \%$, respectively. For clarity of discussion, the prepared PMMA nanocomposites with original graphene loading of $0.1,0.5,1.0$ and $1.5 \mathrm{wt} \%$ were labeled as $\mathrm{C}_{18}$-graphene-0.1/PMMA, $\mathrm{C}_{18}$-graphene-0.5/PMMA, $\mathrm{C}_{18}$-graphene-1.0/PMMA and $\mathrm{C}_{18}$-graphene-1.5/PMMA, respectively. The intrinsic loadings of graphene in these nanocomposites were measured by TGA analysis as discussed in Part 3.3.

\subsection{Characterization}

Fourier Transform Infrared Spectroscopy (FTIR) A Nicolet 6700 Fourier transform infrared spectrometer (Thermo Fisher Scientific, USA) was used for FTIR analysis. FTIR was measured on with scanning from 400 to $4000 \mathrm{~cm}^{-1}$ by using $\mathrm{KBr}$ disks. 
High resolution Transmission Electron Microcopy (HRTEM)

HRTEM was conducted on JEOL JEM -2100 electron microscope (Japan) at an acceleration voltage of $200 \mathrm{kV}$. The sample for HRTEM analysis was prepared by depositing an aliquot of the sample suspension onto a carbon grid and then the solvent was allowed to evaporate.

\section{Scanning Electron Microscopy (SEM)}

The fracture surface of the nanocomposites was observed by FEINova 400 SEM (FEI, Netherlands) with acceleration voltage of $20.0 \mathrm{kV}$. Samples were prepared by immersing the films in liquid nitrogen for $10 \mathrm{~min}$ before fracture. The fracture surfaces were coated with gold before analysis.

\section{$X$-ray Diffraction (XRD)}

XRD measurements were carried out using a Rigaku D/Max-2500 diffractometer (Rigaku, Japan) with $\mathrm{Cu}-\mathrm{K} \alpha$ radiation.

\section{Gel Permeation Chromatograph (GPC)}

GPC measurements were determined using a Waters gel permeation chromatograph (USA) equipped with a Waters 1515 Isocratic HPLC pump, Waters 717 plus Autosampler, three Waters Styragel ${ }^{\circledR}$ HT4 columns collected in series and Waters 2414 Refractive Index Detector refractive index detector. All samples were analyzed at room temperature with tetrahydrofuran as the eluent at the flow rate of $1.0 \mathrm{~mL} / \mathrm{min}$.

\section{Differential Scanning Calorimetry (DSC)}

The glass transition temperature $\left(T_{\mathrm{g}}\right)$ was evaluated by using DSC method. DSC measurements were conducted on TA Q200 (TA, USA) with an approximately $5 \mathrm{mg}$ of each sample in an atmosphere of nitrogen. The samples were tested from $-40^{\circ} \mathrm{Cand}$ then heated to $220^{\circ} \mathrm{C}$ at a heating rate of $10^{\circ} \mathrm{C} / \mathrm{min}$, kept for 5 min to eliminate the thermal history, then cooled to $-40^{\circ} \mathrm{C}$ at a cooling rate of $10^{\circ} \mathrm{C} / \mathrm{min}$. The DSC data reported were taken from the second scan and $T_{\mathrm{g}}$ were calculated.

\section{Thermogravimetric Analysis (TGA)}

TGA and the derivative of TG curves (DTG) were performed with Netzsch STA 449C thermogravimetric analyzer (Germany) under nitrogen atmosphere at a heating rate of $10^{\circ} \mathrm{C} / \mathrm{min}$ and approximately $10 \mathrm{mg}$ of each sample were measured under $\mathrm{N}_{2}$.

\section{Mechanical properties}

The mechanical properties of graphene/PMMA nanocomposites were measured by a universal tensile testing machine (Sans Co. Ltd., China) at room temperature according to ASTM D882-2009. The samples used for mechanical test were prepared by injection molding and conducted with a crosshead speed of $5 \mathrm{~mm} / \mathrm{min}$. In all cases, more than five samples were tested and standard deviations were calculated.

\section{Results and discussion \\ 3.1. Preparation of $\mathrm{C}_{18}$-graphene and its dispersion in MMA}

It is widely known that the maximum improvements in final properties of GPNs can only be achieved when graphene is homogeneously dispersed in the matrix and the external load is efficiently transferred through strong filler/polymer interfacial interaction [13-15]. Thus, how to chemically modify the surface of graphene and design facile preparation route may be essential to generate GPNs with enhanced reinforcement efficiency of graphene. The acylation reaction is one of the most common approaches used for linking molecular moieties onto oxygenated groups alongside the graphene nanosheets [4]. Even if strong reducing reagents are used in chemical deoxygenation of GO and most of oxygen-containing functional groups are removed from the graphene surface, some residual functional groups such as carboxylic and hydroxyl group remain within the graphene sheets, which facilitates further covalent functionalization opening plenty of opportunities for the preparation of GPNs $[17,22$, 50]. In this work, chemically reduced graphene (CRG) was anchored by long alkyl chain via an amidation reaction to enhance the compatibility between the nanosheets and polymer matrix. The amidation reaction was achieved by the DCC-activated coupling reaction between carboxylic moieties on the CRG nanosheets and the amine group of ODA molecule, which was confirmed by FTIR. As presented in Figure 1a, there were some characteristic absorptions of graphene around $1580 \mathrm{~cm}^{-1}$, attributed to $\mathrm{C}=\mathrm{C}$ vibrations of graphene sheets. Moreover, a tiny absorption peak at $1730 \mathrm{~cm}^{-1}$ assigned to the 


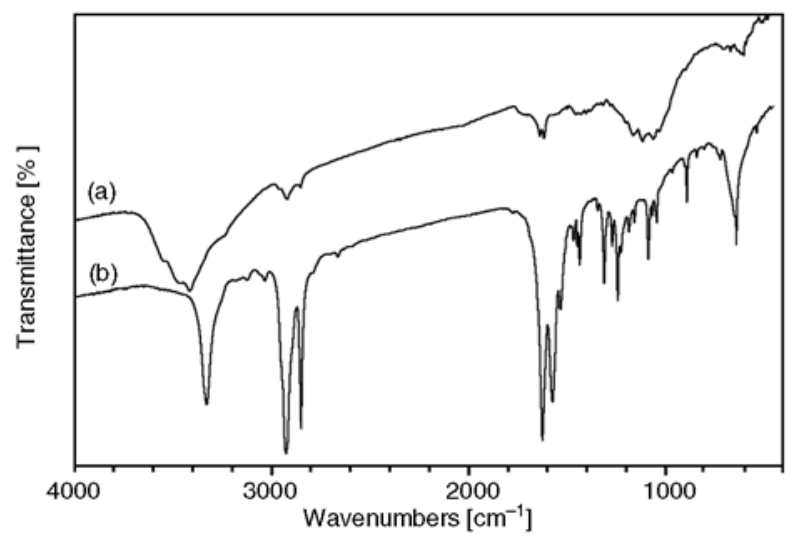

Figure 1. FTIR spectra of CRG (a) and $\mathrm{C}_{18}$-graphene (b)

$\mathrm{C}=\mathrm{O}$ situated at edges of $\mathrm{CRG}$ sheets was also identified. After the reaction with ODA, however, the peak of $\mathrm{C}=\mathrm{O}$ became weaker and new bands around 3300 and $1640 \mathrm{~cm}^{-1}$ due to the formation of amide were observed (Figure 1b). Besides, the absorption peak at 2920 and 2850 (C-H stretch of alkyl chain), $1530 \mathrm{~cm}^{-1}(\mathrm{~N}-\mathrm{H}$ bending of amide) also appeared in the spectrum of $\mathrm{C}_{18}$-graphene. These results confirmed the success of the amidation reaction.

Grafting of long alkyl chain on the nanosheets imparted the functionalized $\mathrm{C}_{18}$-graphene organophilic and was expected to have enhanced solubility in vavious organic medium [48-53]. As shown in Figure 2a, after removal of any unexfoliated agglomerate by ultrasonication and centrifugation, the functionalized $\mathrm{C}_{18}$-graphene was homogenously dispersed in MMA solution. Compared to pure MMA, where the solution was almost clear, the MMA solution containing $\mathrm{C}_{18}$-graphene was blacker, indicating that $\mathrm{C}_{18}$-graphene was more soluble in MMA. Moreover, the blacker MMA solution was very sta- ble and no sedimentation was observed for at least four weeks. To evaluate the degree of exfoliation of organophilic $\mathrm{C}_{18}$-graphene into individual nanosheets in the MMA system, a suspension prepared from original loading of $0.5 \mathrm{wt} \%$ of $\mathrm{C}_{18}$-graphene was examined by High resolution Transmission Electron Microcopy (HRTEM). As seen from Figures $2 b$ and $2 \mathrm{c}$, although some monolayer nanosheets could be detected on the substrate, large quantities of fewlayers smooth graphene ( $<10$ layers) with no significant folding or overlapping were obviously identified, showing that the long alkyl chain present on the sides of the sheets could effectively prevent the restacking of the nanosheets and good dispersibility was achieved in MMA solution.

\subsection{Preparation and microstructure of $\mathrm{C}_{18}$-graphene/PMMA nanocomposites}

Bulk polymerization is ready to prepare PMMA with high molecular weight. After polymerization, the resulting $\mathrm{C}_{18}$-graphene/PMMA nanocomposites darkened to a uniform black as the $\mathrm{C}_{18}$-graphene content increased, contrary to colorless pure PMMA. It has been shown that gelation time was affected by some nanofillers in radical bulk polymerization of MMA and subsequent molecular weight and molecular weight distribution of the nanocomposites were different from those obtained from bulk polymerization of pure MMA [54, 55].

In order to investigate whether similar phenomenon occurred in our research, the molecular weight and polydispersity index of the neat PMMA and $\mathrm{C}_{18^{-}}$ graphene/PMMA nanocomposites with various graphene contents were examined by gel perme-

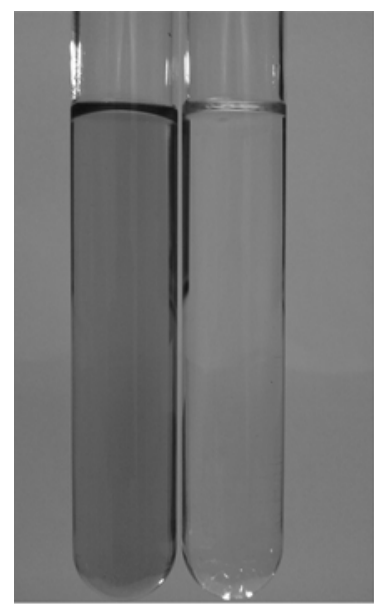

a)

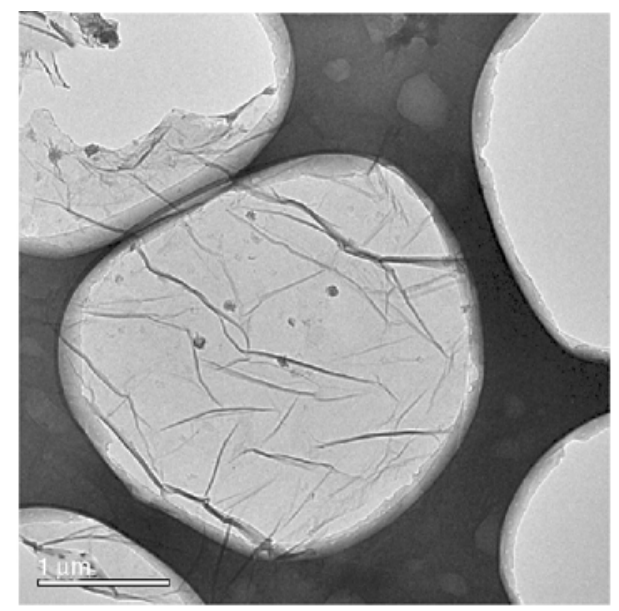

b)

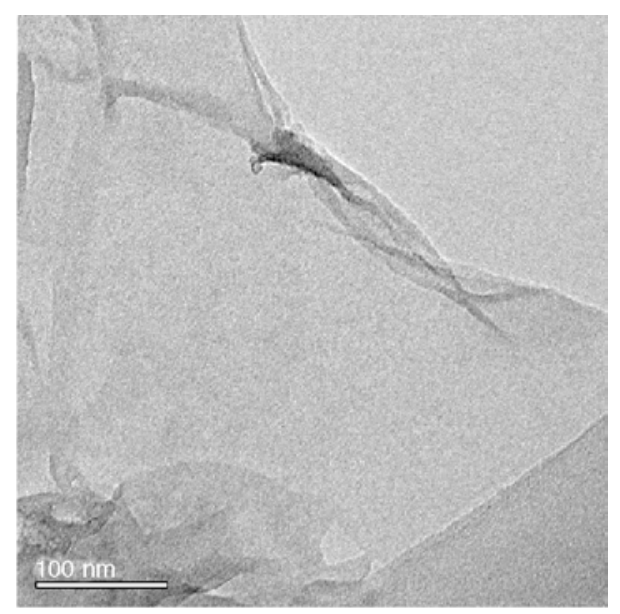

c)

Figure 2. (a) Morphological image of MMA solution containing $\mathrm{C}_{18}$-graphene (left) and pure MMA (right); (b) and (c) HRTEM images of $\mathrm{C}_{18}$-graphene nanosheets in MMA solution of original loading of $0.5 \mathrm{wt} \%$ 
Table 1. Results of GPC analysis for neat PMMA and $\mathrm{C}_{18}$ graphene/PMMA nanocomposites with various graphene contents

\begin{tabular}{|c|c|c|}
\hline Sample & $\begin{array}{c}M_{w}\left(\cdot 10^{5}\right) \\
{[g / m o l]}\end{array}$ & $\begin{array}{c}\mathbf{M}_{\mathbf{w}} / \mathbf{M}_{\mathbf{n}} \\
{[-]}\end{array}$ \\
\hline Neat PMMA & 2.83 & 5.25 \\
\hline $\mathrm{C}_{18}$-graphene-0.1/PMMA & 3.01 & 6.53 \\
\hline $\mathrm{C}_{18}$-graphene-0.5/PMMA & 2.94 & 5.31 \\
\hline $\mathrm{C}_{18}$-graphene-1.0/PMMA & 3.29 & 5.94 \\
\hline $\mathrm{C}_{18}$-graphene-1.5/PMMA & 3.46 & 6.11 \\
\hline
\end{tabular}

ation chromatograph (GPC). Prior to molecular weight measurement, free PMMA was separated from $\mathrm{C}_{18}$-graphene/PMMA nanocomposites by Soxhlet extraction with boiling acetone for $48 \mathrm{~h}$, precipitated in methanol, and finally dried in vacuum at $60^{\circ} \mathrm{C}$ for $48 \mathrm{~h}$. The results of GPC analysis are summarized in Table 1. It could be obviously that PMMA in these composites had higher molecular weight than their neat counterpart and the molecular weight increased slightly as the original loading of $\mathrm{C}_{18^{-}}$ graphene increased. This maybe attributed to the participation of modified graphene nanosheets in the polymerization reaction and the additional consumption of radicals captured by the graphene surface, which was similar to the case where carbon nanotube was used as the nanofiller [54]. In fact, the polymer chains of PMMA nanocomposites measured in GPC were not directly attached to graphene surface because these nanofiller were filtered out prior to the GPC measurements. But this kind of end-tethered PMMA on graphene surface could affect some properties of the resulting GPNs.

The homogeneous dispersion of the nanosheets in the matrix is very crucial to improve the final properties of the GPNs. Li et al. [56] recently reported that simultaneous surface functionalization and reduction of GO was achieved by simple refluxing of GO with octadecylamine (ODA) without the use of any reducing agents and the ODA-modified graphene nanosheets could impove the nanofiller dispersion and thus the conductivity of polymer nanocomposites. To well-disperse the graphene sheets into the polymer, in this study, the as-prepared organophilic $\mathrm{C}_{18}$-graphene was exfoliated into MMA solution via ultrasonication to form a stable colloid and $\mathrm{C}_{18}$-graphene/PMMA nanocomposites were readily obtained by subsequent facile radical-bulk polymerization. To observe the dispersion of graphene in these nanocomposites, X-ray

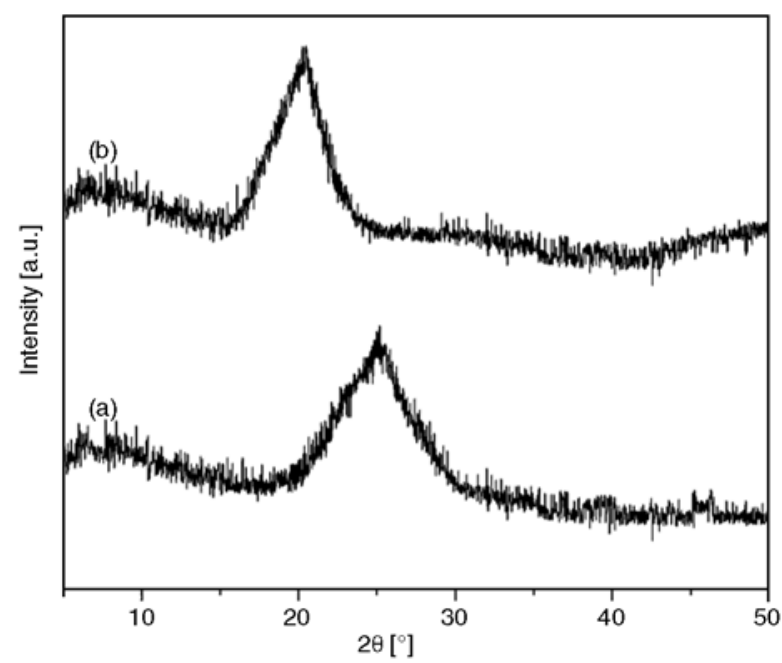

Figure 3. XRD patterns of CRG (a) and $\mathrm{C}_{18}$-graphene (b)

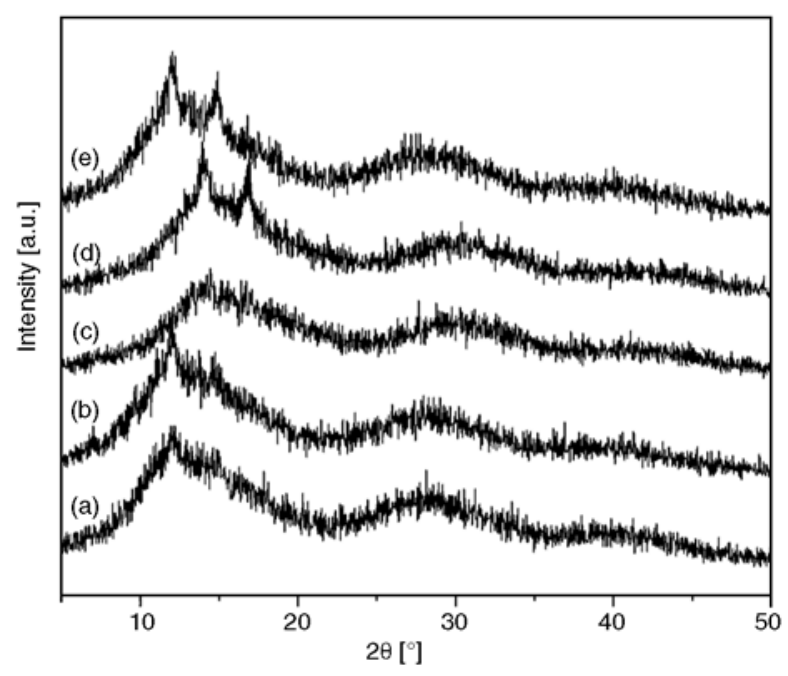

Figure 4. XRD patterns of (a) PMMA and $\mathrm{C}_{18}$-graphene/ PMMA nanocomposites, (b) $\mathrm{C}_{18}$-graphene-0.1/ PMMA, (c) $\mathrm{C}_{18}$-graphene-0.5/PMMA, (d) $\mathrm{C}_{18^{-}}$ graphene-1.0/PMMA and (e) $\mathrm{C}_{18}$-graphene-1.5/ PMMA

Diffraction (XRD) and Scanning Electron Microscopy (SEM) were employed to probe the morphology and nanostructure of these products. Figures 3 and 4 show the XRD patterns of the $\mathrm{CRG}, \mathrm{C}_{18^{-}}$ graphene and PMMA nanocomposites with different loading of $\mathrm{C}_{18}$-graphene, respectively. The $\mathrm{CRG}$ nanosheets showed a (002) diffraction peak at $2 \theta=$ $25^{\circ}$ (Figure $3 \mathrm{a}$ ), which corresponded to a $d$-spacing of $0.35 \mathrm{~nm}$ slightly larger than that of pristine graphite (0.34 nm, JCPDS No. 75-1621). Upon functionalizing with ODA, the strongest diffraction peak of the $\mathrm{C}_{18}$-graphene shifted to a smaller $2 \theta=$ $21^{\circ}$ (Figure $3 \mathrm{~b}$ ), corresponding to a $d$-spacing of $0.41 \mathrm{~nm}$, which indicated that attachment of long alkyl chain along the edges of the nanosheets 


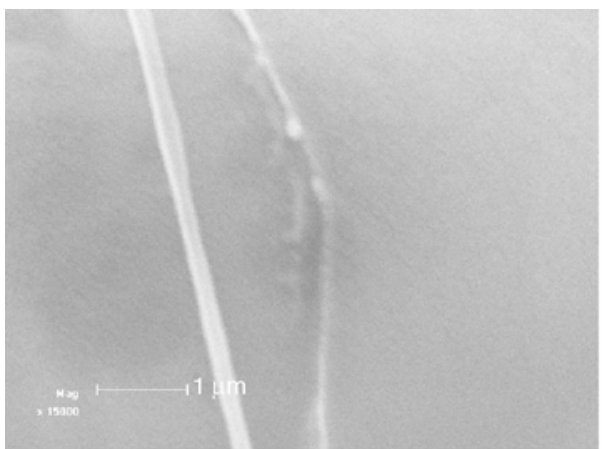

a)

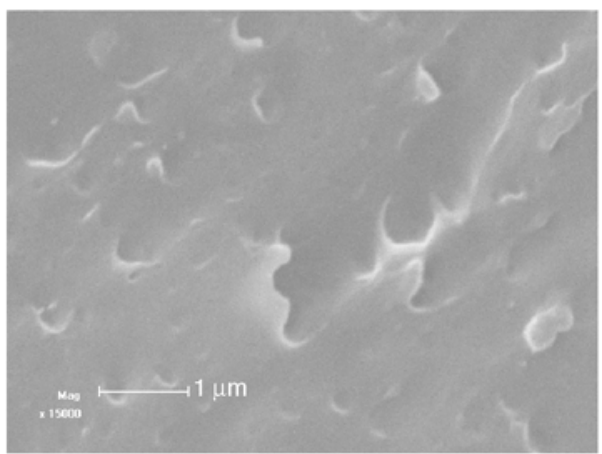

c)

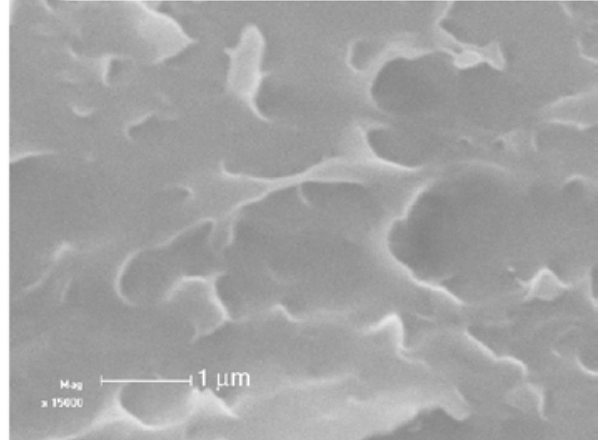

b)

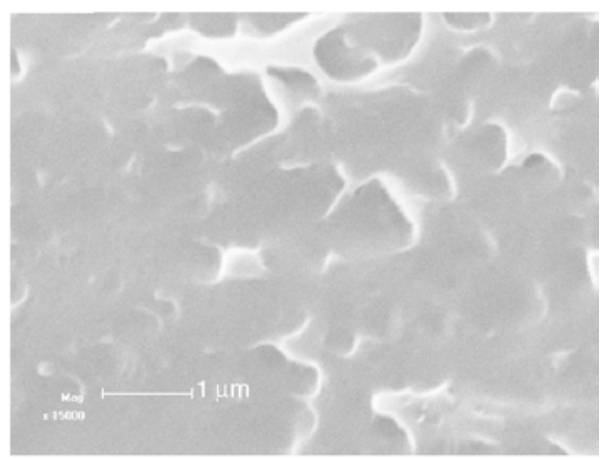

d)

Figure 5. SEM image of cross-section fractured surface of $\mathrm{C}_{18}$-graphene/PMMA nanocomposites. (a) $\mathrm{C}_{18}$-graphene0.1/PMMA, (b) $\mathrm{C}_{18}$-graphene-0.5/PMMA, (c) $\mathrm{C}_{18}$-graphene-1.0/PMMA and (d) $\mathrm{C}_{18}$-graphene-1.5/PMMA.

enlarged the interlayer of $\mathrm{C}_{18}$-graphene and thus would favor the intercalation of monomer and subsequent bulk polymerization within the gallery. For PMMA and $\mathrm{C}_{18}$-graphene/ PMMA nanocomposites, pure PMMA showed a (002) diffraction peak at $2 \theta \approx$ 12 and $30^{\circ}$ (Figure 4a). However, as dispersing the functional $\mathrm{C}_{18}$-graphene nanosheets into the PMMA matrix, the peak of $\mathrm{C}_{18}$-graphene around $21^{\circ}$ (Figure 3b) disappeared in the nanocomposites and only diffraction peaks around 12 and $30^{\circ}$ arisen from PMMA were detected (Figure $4 \mathrm{~b} \sim \mathrm{d}$ ), suggesting that penetration of PMMA chains into inter-layers of graphene were achieved [57], which was furthered confirmed by SEM. Figure 5 shows the cross-section SEM images of the fractured surface of $\mathrm{C}_{18}$-graphene/PMMA nanocomposites. As can be seen from Figure 5, the graphene nanosheets were randomly dispersed in the matrix, especially in the case of lower loadings, showing typical characteristics of good compatibility between the nanosheets and polymer matrix. From Figure 5d, there was clear and irregular entanglement of nanosheets in higher graphene content. Meanwhile, the wrinkled and crumpled profile of the functional nanofiller in the nanocomposites was also detected, which could be largely attributed to strong covalent interaction between graphene nanosheet and PMMA matrix. This phenomenon was also mentioned elsewhere $[44,58,59]$ and should contribute to enhance the final properties of graphene/PMMA nanocomposites.

\subsection{Thermal and mechanical properties of graphene/PMMA nanocomposites}

It is expected that thermal and mechanical properties of the as-prepared $\mathrm{C}_{18}$-graphene/PMMA nanocomposites can be significantly enhanced, largely by the large interfacial area and high aspect ratio of the nanosheet, the homogeneous dispersion of the graphene sheets in the matrix and strong adhesion between graphene and the matrix. Figure 6 displays TGA and corresponding differential thermogravimetric (DTG) thermograms for neat PMMA and its nanocomposites with different graphene loadings under nitrogen atmosphere. The onset temperature of decomposition $\left(T_{\mathrm{d}}\right)$ can be considered as the temperature at the $5 \%$ weight loss. The relative thermal stability of the samples is evaluated by $T_{\mathrm{d}}$ and the temperature of the maximum degradation rate $\left(T_{\max }\right)$ obtained from DTG. These data, providing the information on the thermal degradation behavior, are also listed in Table 2. Both virgin PMMA 
Table 2. Thermal data of PMMA and $\mathrm{C}_{18}$-graphene/PMMA nanocomposites under nitrogen atmosphere

\begin{tabular}{|c|c|c|c|c|}
\hline Sample & $\begin{array}{l}\mathbf{T}_{\mathbf{d}^{\mathbf{a}}} \\
{\left[{ }^{\circ} \mathbf{C}\right]}\end{array}$ & $\begin{array}{l}\mathbf{T}_{\max }{ }^{\mathbf{b}} \\
{\left[{ }^{\circ} \mathbf{C}\right]}\end{array}$ & $\begin{array}{c}\text { Calculated } \\
\text { loading }^{c}\end{array}$ & $\begin{array}{c}\mathbf{T}_{\mathrm{g}} \\
{\left[{ }^{\circ} \mathbf{C}\right]}\end{array}$ \\
\hline PMMA & 249 & 369 & $-^{\mathrm{d}}$ & 107 \\
\hline $\mathrm{C}_{18}$-graphene-0.1/PMMA & 276 & 372 & $0.07 \%$ & 122 \\
\hline $\mathrm{C}_{18}$-graphene-0.5/PMMA & 256 & 370 & $0.21 \%$ & 121 \\
\hline $\mathrm{C}_{18}$-graphene-1.0/PMMA & 273 & 367 & $0.28 \%$ & $-^{\mathrm{d}}$ \\
\hline $\mathrm{C}_{18}$-graphene-1.5/PMMA & 281 & 371 & $0.50 \%$ & 122 \\
\hline
\end{tabular}

${ }^{\mathrm{a}} T_{\mathrm{d}}$ :the degradation temperature at the $5 \%$ weight loss in the decomposition stage

${ }^{\mathrm{b}} T_{\max }$ : the maximum degradation temperature in the decomposition stage

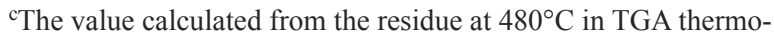
gram of $\mathrm{C}_{18}$-graphene/PMMA nanocomposites

dnot determined

and its nanocomposites exhibited similar weight loss curve profile with one-step threshold in their thermograms. With an onset of thermal degradation at ca. $249^{\circ} \mathrm{C}$, pure PMMA showed mass loss between 300 and $400^{\circ} \mathrm{C}$ and almost no residue left behind above $480^{\circ} \mathrm{C}$. Compared to that of pure PMMA, the TGA curves of $\mathrm{C}_{18}$-graphene/PMMA nanocomposites were shifted toward higher temperature and all the $T_{\mathrm{d}}$ temperatures of these nanocomposites were significantly enhanced with low graphene loadings. For example, $T_{\mathrm{d}}$ values were raised from $249^{\circ} \mathrm{C}$ for virgin PMMA to $276^{\circ} \mathrm{C}$ for $\mathrm{C}_{18}$-graphene-0.1/ PMMA, $256^{\circ} \mathrm{C}$ for $\mathrm{C}_{18}$-graphene-0.5/PMMA, $273^{\circ} \mathrm{C}$ for $\mathrm{C}_{18}$-graphene-1.0/PMMA and $281^{\circ} \mathrm{C}$ for $\mathrm{C}_{18^{-}}$ graphene-1.5/PMMA. Assuming that graphene was very stable against heat and the weight loss that occurred during thermal decomposition of these PMMA nanocomposites in higher temperature $\left(\sim 500^{\circ} \mathrm{C}\right)$ was entirely due to the removal of PMMA entity [30], the residue weight implied the intrinsic loading of graphene in these products, which was also presented in Table 2. With regard to the intrinsic loadings of $\mathrm{C}_{18}$-graphene measured by TGA, therefore, dramatic improvement of thermal stability was achieved with very low loading of graphene in this study. From Table 2, only addition of measured loading of $0.07 \mathrm{wt} \%$ graphene afforded $27^{\circ} \mathrm{C}$ increment in $T_{\mathrm{d}}$ of $\mathrm{C}_{18}$-graphene/PMMA nanocomposites. The reason was presumably that the homogeneously distributed CMG nanosheets could avoid heat concentration upon external thermal exposure and strong interaction between PMMA matrix and chemically modified graphene was introduced via in-situ bulk polymerization [34, 37, 41]. However, further increasing the graphene loading did not result
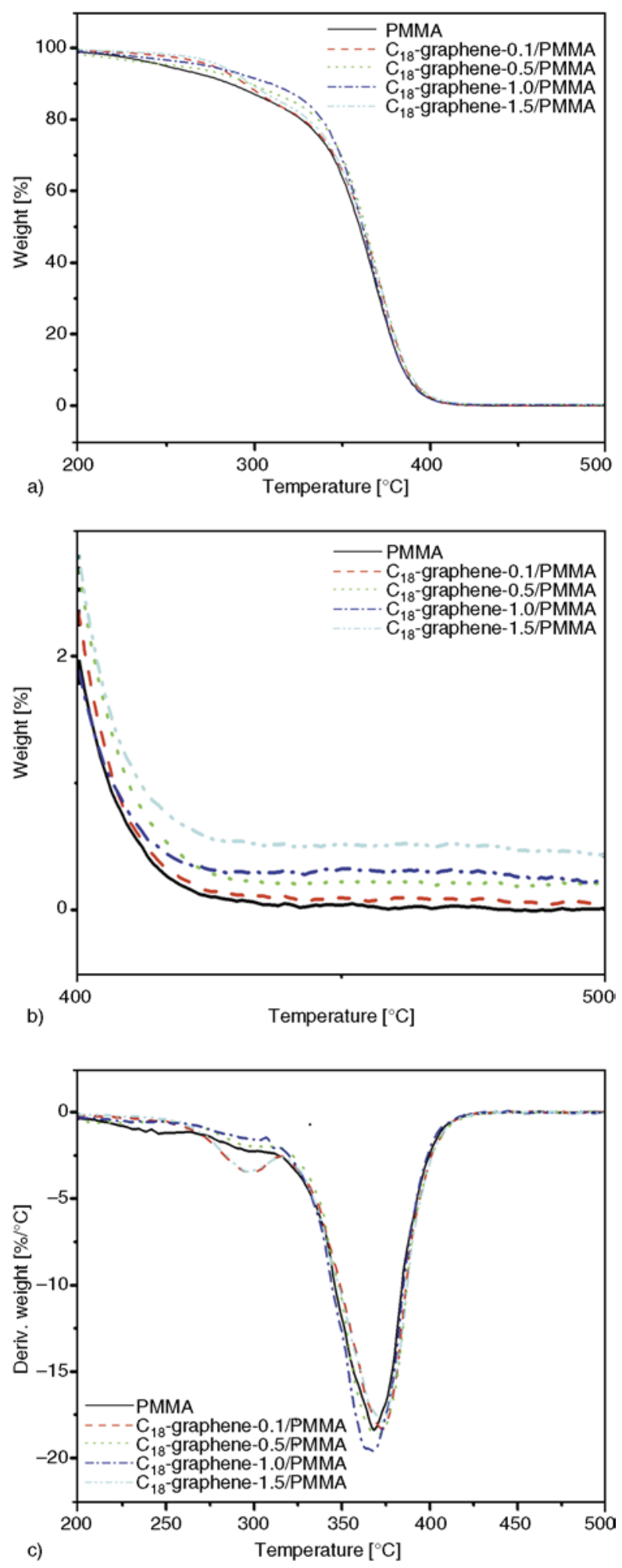

Figure 6. (a) TGA and (b) TGA in the range of $400-500^{\circ} \mathrm{C}$, and (c) DTG thermograms for PMMA and $\mathrm{C}_{18^{-}}$ graphene/PMMA nanocomposites in nitrogen atmosphere

in considerable enhancement of $T_{\mathrm{d}}$. The $T_{\max }$ value was not also affected by the addition of the function- 


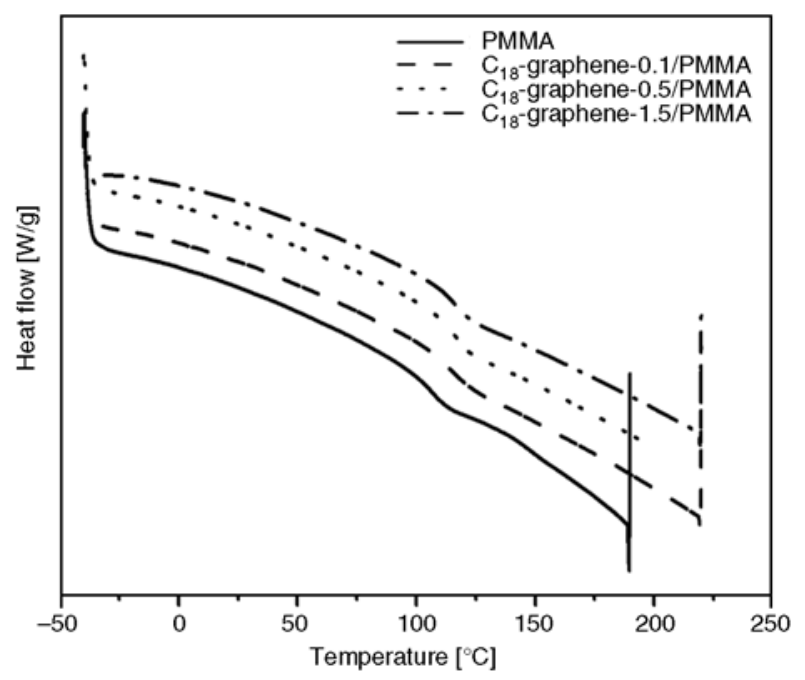

Figure 7. DSC thermograms for PMMA and $\mathrm{C}_{18}$-graphene/ PMMA nanocomposites

alized graphene, showing that the nanofiller did not alter the thermal degradation mechanism.

Figure 7 discloses the DSC thermogram for PMMA and $\mathrm{C}_{18}$-graphene/PMMA nanocomposites and the glass transition temperature $\left(T_{\mathrm{g}}\right)$ is obtained as listed in Table 2. $T_{\mathrm{g}}$ is a macroscopic indication of the segmental relaxation behavior of nanocomposite systems, strongly dependent on embedded nanoparticls. Compared to that of PMMA, $\mathrm{C}_{18}$-graphene/ PMMA nanocomposites showed $15^{\circ} \mathrm{C}$ increase in $T_{\mathrm{g}}$ at an intrinsic loading as low as $0.07 \mathrm{wt} \%$ and little increment was exhibited beyond this loading. Generally, $T_{\mathrm{g}}$ is highly molecular weight dependent, especially below the critical molecular weight of polymer. With regard to the same preparation condition and little difference in molecular weight for all samples in this case, however, the observed increase in $T_{\mathrm{g}}$ could not result largely from the molecular weight of PMMA. Therefore, the substantial enhancement in the $T_{\mathrm{g}}$ of these nanocomposites was attributed to the restriction in chain mobility due to confinement effect of 2D-layered graphene incorporated into the matrix and strong nanofiller-polymer interaction, which was similar to other reports elsewhere [27, 44].

As an important petrochemical polymer used in various fields, it is of much concern about mechanical property of PMMA composites. Figure 8 shows the typical stress-strain curves for pure PMMA and its nanocomposites with different graphene loadings. Taking the intrinsic loading of the nanofiller in these nanocomposites into account, it was obvious

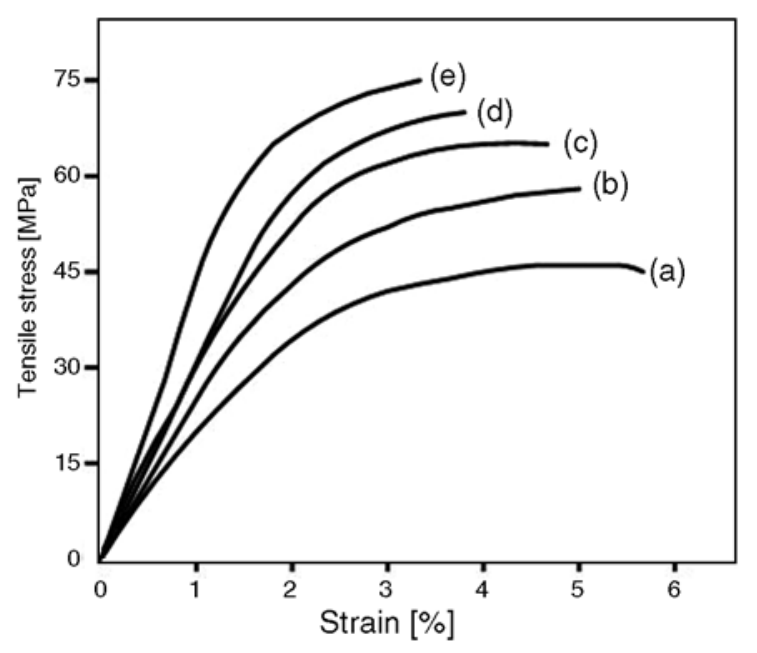

Figure 8. Typical stress-strain curves for (a) pure PMMA and $\mathrm{C}_{18}$-graphene/PMMA nanocomposites,

(b) $\mathrm{C}_{18}$-graphene-0.1/PMMA,

(c) $\mathrm{C}_{18}$-graphene-0.5/ PMMA,

(d) $\mathrm{C}_{18}$-graphene-1.0/PMMA and

(e) $\mathrm{C}_{18}$-graphene-1.5/PMMA

that low loading of graphene into the polymer matrix had a significant influence on the mechanical behavior and the tensile strength of $\mathrm{C}_{18^{-}}$ graphene/PMMA nanocomposites with low graphene loading was dramatically enhanced as compared to that of pure PMMA matrix. For example, with intrinsic graphene loading of only $0.5 \mathrm{wt} \%$ for $\mathrm{C}_{18^{-}}$ graphene-1.5/PMMA (the intrinsic loading of the nanocomposites is listed in Table 2), the tensile strength increased from $45 \mathrm{MPa}$ of pure PMMMA to $75 \mathrm{MPa}$ and the increase was $67 \%$. The increasing trend was especially clear with lower loading. For example, the $0.07 \mathrm{wt} \%$ intrinsic loading of graphene increased the tensile strength up to $57 \mathrm{MPa}$ and the tensile strength changed slightly without a pronounced threshold while further raising graphene loading, Suggesting that there existed a mechanical percolation probably due to the nanosheet restacking in the case of higher graphene content, which was consistent with the other reports [16, 57, 58]. On the contrary, the elongation at break of the nanocomposites gradually decreased with graphene loading in contrast to that of pure PMMA. The value of the elongation at break decreased from $6 \%$ for pure PMMA to $3.5 \%$ for $\mathrm{C}_{18}$-graphene-1.5/ PMMA. The mechanical reinforcement with low graphene loading could be attributed to the homogeneous dispersion of graphene sheets in the matrix and the strong interfacial adhesion between chemi- 
cally modified graphene and PMMA. Because of the high surface area of graphene in the nanocomposites, the applied stress was expected to transfer effectively from the matrix to graphene layers resulting in enhancement of mechanical properties $[59,60]$.

\section{Conclusions}

Chemically modified graphene by long alkyl chain was prepared and the graphene/PMMA nanocomposites were successfully fabricated via a facile in situ bulk polymerization. Thermal and mechanical properties of the nanocomposites were significantly enhanced at very low graphene loadings. The reinforcement with low graphene loading was attributed to homogeneous dispersion of the nanosheets and enhanced nanofiller-matrix interfacial interaction. Because of easy performance of functionalized graphene and bulk polymerization, the facile method presented here could be extended to the implementation of other graphene-based polymer nanocomposites.

\section{Acknowledgements}

The authors would like to gratefully acknowledge the supports by Municipal Science Foundation Project of CQ CSTC (No.2007BB4442) and of CQEC (No.KJ110421) and Open-end Fund of Hi-tech Lab for Mountain Road Construction and Maintenance, CQTJU (CQMRCM-10-5).

\section{References}

[1] Geim A. K., Novoselov K. S.: The rise of graphene. Nature Materials, 6, 183-191 (2007).

DOI: $10.1038 /$ nmat 1849

[2] Geim A. K.: Graphene: Status and prospects. Science, 324, 1530-1534 (2009). DOI: $10.1126 /$ science. 1158877

[3] Allen M. J., Tung V. C., Kaner R. B.: Honeycomb carbon: A review of graphene. Chemical Review, 110, 132-145 (2010). DOI: $10.1021 / \mathrm{cr} 900070 d$

[4] Sun Z., James D. K., Tour J. M.: Graphene chemistry: Synthesis and manipulation. The Journal of Physical Chemistry Letters, 2, 2425-2432 (2011). DOI: $10.1021 / \mathrm{jz201000a}$

[5] Kim J., Kim F., Huang J.: Seeing graphene-based sheets. MaterialsToday, 13, 28-38 (2010). DOI: 10.1016/S1369-7021(10)70031-6

[6] Zhu Y., Murali S., Cai W., Li X., Suk J. W., Potts J. R., Ruoff R. S.: Graphene and graphene oxide: Synthesis, properties, and applications. Advanced Materials, 22, 3906-3924 (2010).

DOI: $10.1002 / \mathrm{adma} .201001068$
[7] Wu J., Pisula W., Müllen K.: Graphenes as potential material for electronics. Chemical Review, 107, 718747 (2007).

DOI: $10.1021 / \mathrm{cr} 068010 \mathrm{r}$

[8] Singh V., Joung D., Zhai L., Das S., Khondaker S. I., Seal S.: Graphene based materials: Past, present and future. Progress in Materials Science, 56, 1178-1271 (2011).

DOI: $10.1016 /$ j.pmatsci.2011.03.003

[9] Stankovich S., Dikin D. A., Dommett G. H. B., Kohlhaas K. M., Zimney E. J., Stach E. A., Piner R. D., Nguyen S. T., Ruoff R. S.: Graphene-based composite materials. Nature, 442, 282-286 (2006).

DOI: 10.1038/nature04969

[10] Shao Y., Wang J., Wu H., Liu J., Aksay I. A., Lin Y.: Graphene based electrochemical sensors and biosensors: A review. Electroanalysis, 22, 1027-1036 (2010). DOI: $10.1002 /$ elan.200900571

[11] Bao Q., Zhang H., Yang J. X., Wang S., Tang D. Y., Jose R., Ramakrishna S., Lim C. T., Loh K. P.: Graphenepolymer nanofiber membrane for ultrafast photonics. Advanced Functional Materials, 20, 782-791 (2010). DOI: $10.1002 / \mathrm{adfm} .200901658$

[12] Zhu Y., Murali S., Stoller M. D., Ganesh K. J., Cai W., Ferreira P. J., Pirkle A., Wallace R. M., Cychosz K. A., Thommes M., Su D., Stach E. A., Ruoff R. S.: Carbonbased supercapacitors produced by activation of graphene. Science, 332, 1537-1541 (2011). DOI: $10.1126 /$ science. 1200770

[13] Kim H., Abdala A. A., Macosko C. W.: Graphene/ polymer nanocomposites. Macromolecules, 43, 65156530 (2010).

DOI: $10.1021 / \mathrm{ma100572 \textrm {e }}$

[14] Kuilla T., Bhadra S., Yao D., Kim N. H., Bose S., Lee J. H.: Recent advances in graphene based polymer composites. Progress in Polymer Science, 35, 1350-1375 (2010).

DOI: $10.1016 /$ j.progpolymsci.2010.07.005

[15] Potts J. R., Dreyer D. R., Bielawski C. W., Ruoff R. S.: Graphene-based polymer nanocomposites. Polymer, 52, 5-25 (2011). DOI: $10.1016 /$ j.polymer.2010.11.042

[16] Rafiee M. A., Rafiee J., Wang Z., Song H., Yu Z-Z., Koratkar N.: Enhanced mechanical properties of nanocomposites at low graphene content. ACS Nano, 3, 3884-3890 (2009).

DOI: $10.1021 / \mathrm{nn} 9010472$

[17] Loh K. P., Bao Q., Ang P. K., Yang J.: The chemistry of graphene. Journal of Materials Chemistry, 20, 22772289 (2010). DOI: $10.1039 / \mathrm{b} 920539 \mathrm{j}$

[18] Park R., Ruoff R. S.: Chemical methods for the production of graphenes. Nature. Nanotechnology, 4, $217-$ 224 (2009).

DOI: $10.1038 /$ nnano.2009.58 
[19] Dreyer D. R., Park S., Bielawski C. W., Ruoff R. S.: The chemistry of graphene oxide. Chemical Society Reviews, 39, 228-240 (2010).

DOI: $10.1039 / \mathrm{b} 917103 \mathrm{~g}$

[20] Salavagione H. J., Gómez M. A., Martínez G.: Polymeric modification of graphene through esterification of graphite oxide and poly(vinyl alcohol). Macromolecules, 42, 6331-6334 (2009).

DOI: $10.1021 / \mathrm{ma} 900845 \mathrm{~W}$

[21] Park S., Dikin D. A., Nguyen S. T., Ruoff R. S.: Graphene oxide sheets chemically cross-linked by polyallylamine. The Journal of Physical Chemistry C, 113, 15801-15804 (2009).

DOI: $10.1021 /$ jp907613s

[22] Hsiao M-C., Liao S-H., Yen M-Y., Liu P-I., Pu N-W., Wang C-A., Ma C-C. M.: Preparation of covalently functionalized graphene using residual oxygen-containing functional groups. ACS Applied Materials and Interfaces, 2, 3092-3099 (2010).

DOI: $10.1021 / \mathrm{am} 100597 \mathrm{~d}$

[23] Salavagione H. J., Martínez G.: Importance of covalent linkages in the preparation of effective reduced graphene oxide-poly(vinyl chloride) nanocomposites. Macromolecules, 44, 2685-2692 (2011). DOI: $10.1021 / \mathrm{ma} 102932 \mathrm{c}$

[24] Sun S., Cao Y., Feng J., Wu P.: Click chemistry as a route for the immobilization of well-defined polystyrene onto graphene sheets. Journal of Materials Chemistry, 20, 5605-5607 (2010). DOI: $10.1039 / \mathrm{c} 0 \mathrm{jm} 01269 \mathrm{f}$

[25] Pan Y., Bao H., Sahoo N. G., Wu T., Li L.: Water-soluble poly( $N$-isopropylacrylamide)-graphene sheets synthesized via click chemistry for drug delivery. Advanced Functional Materials, 21, 2754-2763 (2011). DOI: 10.1002/adfm.201100078

[26] Xu X., Luo Q., Lv W., Dong Y., Lin Y., Yang Q., Shen A., Pang D., Hu J., Qin J., Li Z.: Functionalization of graphene sheets by polyacetylene: Convenient synthesis and enhanced emission. Macromolecular Chemistry Physics, 212, 768-773 (2011).

DOI: $10.1002 /$ macp. 201000608

[27] Pramoda K. P., Hussain H., Koh H. M., Tan H. R., He C. B.: Covalent bonded polymer-graphene nanocomposites. Journal of Polymer Science Part A: Polymer Chemistry, 48, 4262-4267 (2010).

DOI: $10.1002 /$ pola. 24212

[28] Liu J., Yang W., Tao L., Li D., Boyer C., Davis T. P.: Thermosensitive graphene nanocomposites formed using pyrene-terminal polymers made by RAFT polymerization. Journal of Polymer Science Part A: Polymer Chemistry, 48, 425-433 (2010).

DOI: $10.1002 /$ pola. 23802

[29] Gonçalves G., Marques P. A. A. P., Barros-Timmons A., Bdkin I., Singh M. K., Emami N., Grácio J.: Graphene oxide modified with PMMA via ATRP as a reinforcement filler. Journal of Materials Chemistry, 20, 99279934 (2010).

DOI: $10.1039 / \mathrm{c} 0 \mathrm{jm} 01674 \mathrm{~h}$
[30] Fang M., Wang K., Lu H., Yang Y., Nutt S.: Covalent polymer functionalization of graphene nanosheets and mechanical properties of composites. Journal of Materials Chemistry, 19, 7098-7105 (2009).

DOI: 10.1039/b908220d

[31] Deng Y., Li Y., Dai J., Lang M., Huang X.: An efficient way to functionalize graphene sheets with presynthesized polymer via ATNRC chemistry. Journal of Polymer Science Part A: Polymer Chemistry, 49, 15821590 (2011).

DOI: $10.1002 /$ pola.24579

[32] Lee S. H., Dreyer D. R., An J., Velamakanni A., Piner R. D., Park S., Zhu Y., Kim S. O., Bielawski C. W., Ruoff R. S.: Polymer brushes via controlled, surfaceinitiated atom transfer radical polymerization (ATRP) from graphene oxide. Macromolecular Rapid Communications, 31, 281-288 (2010).

DOI: $10.1002 /$ marc.200900641

[33] Lin Y., Jin J., Song M.: Preparation and characterisation of covalent polymer functionalized graphene oxide. Journal of Materials Chemistry, 21, 3455-3461 (2011). DOI: $10.1039 / \mathrm{c} 0 \mathrm{jm} 01859 \mathrm{~g}$

[34] Liang J., Huang Y., Zhang L., Wang Y., Ma Y., Guo T., Chen Y.: Molecular-level dispersion of graphene into poly(vinyl alcohol) and effective reinforcement of their nanocomposites. Advanced Functional Materials, 19, 1-6 (2009). DOI: $10.1002 / \mathrm{adfm} .200801776$

[35] Ramanathan T., Abdala A. A., Stankovich S., Dikin D. A., Herrera-Alonso M., Piner R. D., Adamson D. H., Schniepp H. C., Chen X., Ruoff R. S., Nguyen S. T., Aksay I. A., Prud'homme R. K., Brinson L. C.: Functionalized graphene sheets for polymer nanocomposites. Nature Nanotechnology, 3, 327-331 (2008). DOI: 10.1038/nnano.2008.96

[36] Vickery J. L., Patil A. J., Mann S.: Fabrication of graphene-polymer nanocomposites with higher-order three-dimensional architectures. Advanced Materials, 21, 2180-2184 (2009). DOI: $10.1002 / \mathrm{adma} .200803606$

[37] Kuila T., Bose S., Hong C. E., Uddin M. E., Khanra P., Kim N. H., Lee J. H.: Preparation of functionalized graphene/linear low density polyethylene composites by a solution mixing method. Carbon, 49, 1033-1051 (2011). DOI: $10.1016 /$ j.carbon.2010.10.031

[38] Putz K. W., Compton O. C., Palmeri M. J., Nguyen S. T., Brinson L. C.: High-nanofiller-content graphene oxide-polymer nanocomposites via vacuum-assisted self-assembly. Advanced Functional Materials, 20, 3322-3329 (2010). DOI: $10.1002 / \mathrm{adfm} .201000723$

[39] Zhao X., Zhang Q., Hao Y., Li Y., Fang Y., Chen D.: Alternate multilayer films of poly(vinyl alcohol) and exfoliated graphene oxide fabricated via a facial layerby-layer assembly. Macromolecules, 43, 9411-9416 (2010). DOI: $10.1021 / \mathrm{ma101456y}$ 
[40] Liu J., Tao L., Yang W., Li D., Boyer C., Wuhrer R., Braet F., Davis T. P.: Synthesis, characterization, and multilayer assembly of $\mathrm{pH}$ sensitive graphene-polymer nanocomposites. Langmuir, 26, 10068-10075 (2010).

DOI: $10.1021 / 1 \mathrm{a} 1001978$

[41] Potts J. R., Lee S. H., Alam T. M., An J., Stoller M. D., Piner R. D., Ruoff R. S.: Thermomechanical properties of chemically modified graphene/poly(methyl methacrylate) composites made by in situ polymerization. Carbon, 49, 2615-2623 (2011). DOI: $10.1016 /$ j.carbon.2011.02.023

[42] Oh S. M., Lee H., Jeong H. M., Kim B. K.: The properties of functionalized graphene sheet/poly(ethyl methacrylate) nanocomposites: The effects of preparation method. Macromolecular Research, 19, 379-384 (2011). DOI: $10.1007 / \mathrm{s} 13233-011-0404-0$

[43] Hu H., Wang X., Wang J., Wan L., Liu F., Zheng H., Chen R., Xu C.: Preparation and properties of graphene nanosheets-polystyrene nanocomposites via in situ emulsion polymerization. Chemical Physics Letters, 484, 247-253 (2010).

DOI: $10.1016 /$ j.cplett.2009.11.024

[44] Wang J., Hu H., Wang X., Xu C., Zhang M., Shang X.: Preparation and mechanical and electrical properties of graphene nanosheets-poly(methyl methacrylate) nanocomposites via in situ suspension polymerization. Journal of Applied Polymer Science, 122, 1866-1871 (2011).

DOI: $10.1002 / a p p .34284$

[45] Ray S. S., Okamoto M.: Polymer/layered silicate nanocomposites: A review from preparation to processing. Progress in Polymer Science, 28, 1539-1641 (2003). DOI: 10.1016/j.progpolymsci.2003.08.002

[46] Moniruzzaman M., Winey K. I.: Polymer nanocomposites containing carbon nanotubes. Macromolecules, 39, 5194-5205 (2006).

DOI: $10.1021 / \mathrm{ma} 060733 p$

[47] Hummers W. S., Offeman R. E.: Preparation of graphitic oxide. Journal of the American Chemical Society, 80, 1339 (1958). DOI: $10.1021 / \mathrm{ja} 01539 \mathrm{a} 017$

[48] Cao Y., Feng J., Wu P.: Alkyl-functionalized graphene nanosheets with improved lipophilicity. Carbon, 48, 1683-1685 (2010).

DOI: 10.1016/j.carbon.2009.12.061

[49] Stankovich S., Dikin D. A., Piner R. D., Kohlhaas K. A., Kleinhammes A., Jia Y., Wu Y., Nguyen S. T., Ruoff R. S.: Synthesis of graphene-based nanosheets via chemical reduction of exfoliated graphite oxide. Carbon, 45, 1558-1565 (2007). DOI: $10.1016 /$ j.carbon.2007.02.034
[50] Yuan X-Y.: Progress in preparation of graphene. Journal of Inorganic Materials, 26, 561-570 (2011).

DOI: 10.3724/SP.J.1077.2011.00561

[51] Wang G., Shen X., Wang B., Yao J., Park J.: Synthesis and characterisation of hydrophilic and organophilic graphene nanosheets. Carbon, 47, 1359-1364 (2009). DOI: $10.1016 /$ j.carbon.2009.01.027

[52] Niyogi S., Bekyarova E., Itkis M. E., Mcwilliams J. L., Hamon M. A., Haddon R. C.: Solution properties of graphite and graphene. Journal of the American Chemical Society, 128, 7720-7721 (2006).

DOI: $10.1021 / \mathrm{ja} 060680 \mathrm{r}$

[53] Tessonnier J-P., Barteau M. A.: Dispersion of alkylchain-functionalized reduced graphene oxide sheets in nonpolar solvents. Langmuir, 28, 6691-6697 (2012). DOI: $10.1021 / 1 \mathrm{a} 2051614$

[54] Park S. J., Cho M. S., Lim S. T., Choi H. J., Jhon M. S.: Synthesis and dispersion characteristics of multiwalled carbon nanotube composites with poly(methyl methacrylate) prepared by in-situ bulk polymerization. Macromolecular Rapid Communications, 24, 10701073 (2003). DOI: $10.1002 /$ marc.200300089

[55] Qu X., Guan T., Liu G., She Q., Zhang L.: Preparation, structural characterization, and properties of poly (methyl methacrylate)/montmorillonite nanocomposites by bulk polymerization. Journal of Applied Polymer Science, 97, 348-357 (2005).

DOI: $10.1002 / a p p .21749$

[56] Li W., Tang X-Z., Zhang H-B., Jiang Z-G., Yu Z-Z., Du X-S., Mai Y-W.: Simultaneous surface functionalization and reduction of graphene oxide with octadecylamine for electrically conductive polystyrene composites. Carbon, 49, 4724-4730 (2011). DOI: $10.1016 /$ j.carbon.2011.06.077

[57] Zhao X., Zhang Q., Chen D., Lu P.: Enhanced mechanical properties of graphene-based poly(vinyl alcohol) composites. Macromolecules, 43, 2357-2363 (2010). DOI: $10.1021 / \mathrm{ma} 902862 \mathrm{u}$

[58] Yuan X.: Enhanced interfacial interaction for effective reinforcement of poly(vinyl alcohol) nanocomposites at low loading of graphene. Polymer Bulletin, 67, 17851797 (2011).

DOI: $10.1007 / \mathrm{s} 00289-011-0506-\mathrm{Z}$

[59] Salavagione H. J., Martínez G., Ellis G.: Recent advances in the covalent modification of graphene with polymers. Macromolecular Rapid Communications, 32, 1771-1789 (2011). DOI: $10.1002 /$ marc.201100527

[60] Gong L., Kinloch I. A., Young R. J., Riaz I., Jalil R., Novoselov K. S.: Interfacial stress transfer in a graphene monolayer nanocomposite. Advanced Materials, 22, 2694-2697 (2010). DOI: $10.1002 / \mathrm{adma} .200904264$ 